\title{
Determinação da cinética de secagem de produtos alimentícios usando algoritmos genéticos
}

\author{
Thaís Alves Barbosa', Fran Sérgio Lobato² \\ ${ }^{1}$ Instituto Federal Goiano - IF Goiano, Campus Morrinhos, Morrinhos, Goiás, Brasil. E-mail: thais.barbosa@ifgoiano.edu.br \\ ${ }^{2}$ Universidade Federal de Uberlândia - UFU, Campus Santa Mônica, Uberlândia, Minas Gerais, Brasil. E-mail: fslobato@ufu.br
}

Recebido: 15/04/2016; Aceito: 07/07/2016.

\section{RESUMO}

A secagem de produtos alimentícios consiste na redução do seu volume e de sua massa durante o processo de desidratação que ocorre através da remoção de água. Esta operação unitária é amplamente utilizada na indústria com o objetivo de obter um produto com determinadas especificações de mercado. Entre as variáveis que mais influenciam este fenômeno, pode-se citar o tempo e a temperatura. Matematicamente, o estudo da cinética de secagem de produtos alimentícios é realizado considerando a determinação de parâmetros que caracterizam a cinética de secagem. Neste trabalho é formulado e resolvido um problema de otimização que consiste da minimização da diferença entre os dados experimentais e o calculado usando diferentes modelos matemáticos, empregados para estudar as características de secagem de dois produtos alimentícios, a saber, abacaxi e banana usando Algoritmos Genéticos. Foram determinadas quatro equações empíricas para a cinética de secagem de cada um dos produtos estudados. Os resultados obtidos demonstraram que a metodologia proposta configurou-se como uma interessante abordagem para a estimação de parâmetros.

Palavras-chave: frutas desidratadas, equações empíricas, estimação de parâmetros, otimização.

\section{Determination of drying kinetics of food products using genetic algorithms}

\begin{abstract}
Drying of food products consists of mass and volume reduction during the dehydration process through water removal. This unitary operation is widely used in industries to obtain a product with determined market specifications. Among the variables that most affect this phenomenon we can cite the time and temperature. Mathematically, the study of drying kinetics of food products is realized considering the determination of parameters in drying kinetics. In this contribution is formulated and solved an optimization problem that consists of minimization of difference between experimental data and computed values using different mathematical models to study the drying characteristic of pineapple and banana during thin-layer drying using Genetic Algorithms. Four empirical equations to estimate the drying kinetics were determined. The results obtained demonstrated that the proposed methodology is configured as an interesting approach to parameters estimation.
\end{abstract}

Key words: dehydrated fruits, empirical equation, parameter estimation, optimization. 


\section{Introdução}

A secagem ou desidratação configura-se como um dos processos de engenharia mais utilizados para a conservação de produtos alimentícios, já que a grande maioria destes sofre deterioração com a ação microbiana (TADINI et al., 2016). Esta operação unitária é responsável por transferir a umidade que está em um sólido para uma fase gasosa não saturada de forma a adequar o produto a uma determinada especificação de mercado (LOBATO et al., 2008, ARRUDA et al., 2009). Dentre as vantagens desta operação unitária pode-se citar: a conservação do produto por um período de tempo maior do que aquele requerido pelo produto in natura, a redução do seu peso (redução do custo de transporte e armazenamento) e a capacidade de conservação das características físicas e nutritivas (LOBATO et al., 2008, ARRUDA et al., 2009).

A secagem é um assunto abrangente e de alta complexidade, pois envolve trocas simultâneas de calor, massa e momento. Vários parâmetros afetam o processo de secagem, sendo que muitos deles são dependentes da estrutura do sólido e podem apresentar variações para um mesmo produto que tenha sido feito por processos diferentes ou até mesmo em lotes diferentes do mesmo processo (LOBATO et al., 2008, ARRUDA et al., 2009).

Apesar de muitas teorias terem sido publicadas ao longo dos anos, a falta de comparação com resultados experimentais tem levado os fabricantes de secadores industriais a optarem pelo emprego de métodos de projeto empíricos baseados em testes em plantas pilotos para scale-up. Experiências mal sucedidas de aplicações dessas teorias no projeto de secadores ocorreram mediante algumas considerações errôneas no desenvolvimento do modelo, aliado ao grande número de parâmetros requeridos, os quais são difíceis de serem medidos e podem levar a erros cumulativos (KEMP; OAKLEY, 2002).

A modelagem matemática do fenômeno de secagem é regida por um sistema de equações diferenciais que representam os balanços de massa, energia e quantidade de movimento. Associado a estes modelos fenomenológicos, uma série de equações empíricas (constitutivas) devem ser empregadas para que esse fenômeno altamente não linear possa ser representado. Dentre as equações empíricas que devem ser determinadas, expresses para a umidade de equilíbrio e para a cinética de secagem estão entre as mais importantes. Cabe ressaltar que estes modelos isoladamente não são capazes de descrever o processo de transferência de calor e massa em camadas espessas, uma vez que os balanços de massa e energia da fase gasosa não são considerados. Entretanto, estes estudos são indispensáveis na predição dos fenômenos de transferência de massa e calor (ARRUDA et al., 2009).

Em se tratando da cinética de secagem, a literatura especializada apresenta uma série de modelos com aplicações em diferentes áreas da ciência e da engenharia. Dentre estes pode-se citar o estudo da cinética de secagem de: $i$ ) grãos de soja (OVERHULTS et al., 1973; WHITE et al., 1978); ii) frutas (CEYLAN et al., 2007; SHAHARI, 2012); iii) fertilizantes (LOBATO et al., 2008, ARRUDA et al., 2009); iv) sementes de crambe (FARIA et al., 2012); v) bananas (KUMAR et al., 2012; MONTEIRO et al., 2016); vi) maçãs (ZLATANOVIC et al., 2013); vii) mamão (UDOMKUN et al., 2015) e ix) batatas e maçãs (SINGH et al., 2014). Em cada um destes modelos, a determinação dos parâmetros que caracterizam a cinética de secagem é obtida através da formulação e resolução de um problema de otimização, que consiste na obtenção dos melhores parâmetros que minimizam o somatório dos desvios quadráticos entre o modelo proposto e os pontos experimentais.

Tradicionalmente, este problema têm sido tratado através da aplicação de técnicas clássicas, isto é, metodologias que fazem uso de informações sobre o gradiente da função objetivo e das restrições para a atualização do candidato à solução do problema. Nos últimos anos, as técnicas evolutivas têm sido empregadas para a resolução dos problemas de otimização. Isto se deve, entre outros aspectos, a habilidade que estes apresentam para escapar de ótimos locais, pela facilidade encontrada no tratamento de problemas com variáveis mistas (inteiras + contínuas + discretas) e em problemas com restrições de diferentes naturezas (SARAMAGO, 1999).

Esta contribuição tem por objetivo determinar os parâmetros das equações empíricas da cinética de secagem do abacaxi e da banana considerando diferentes modelos descritos na literatura. Para resolver este problema de otimização será utilizado os tradicionais Algoritmos Genéticos (MICHALEWICZ, 1996; SARAMAGO, 1999). Este trabalho está estruturado como segue: a seção 2 apresenta a descrição dos materiais e métodos empregados neste trabalho. Esta contém a descrição do procedimento experimental, os modelos matemáticos utilizados para a caracterização da cinética de secagem, a formulação matemática do problema de otimização, bem como a metodologia para avaliar a qualidade do ajuste e uma breve descrição dos Algoritmos Genéticos, metodologia empregada para a resolução do problema de otimização proposto. $\mathrm{Na}$ seção 3 são apresentados os resultados obtidos com a aplicação da metodologia proposta. Finalmente, as conclusões e perspectivas são apresentadas na última seção. 


\section{Material e Métodos}

$\mathrm{O}$ estudo foi realizado no Laboratório de Agroindústria do Instituto Federal Goiano, Campus Morrinhos-GO. Como matéria-prima utilizou-se o abacaxi pérola (Ananascomosus L. Merril) e banana maçã (Musa acuminata). Antes do processo de desidratação foram realizadas algumas etapas: com o objetivo de melhorar a aparência e qualidade do produto, além de prolongar a vida útil do mesmo:

$\mathrm{Na}$ higienização e sanitização: as frutas foram lavadas com sabão neutro a fim de eliminar as sujidades físicas contidas na casca e, posteriormente, mergulhadas em solução de hipoclorito de sódio, por dez minutos. Após a sanitização as frutas foram descascadas e cortadas em rodelas. O formato do corte é opcional, desde que se mantenha maior uniformidade possível. No caso do abacaxi, foi retirado o miolo. Finalizado o processo de corte as frutas passam por um branqueamento, que é um tratamento térmico preliminar à desidratação. Este visa remover gases, inativar enzimas, fixar cor e textura, além de reduzir a quantidade de microrganismos na superfície do produto. Neste processo, as frutas foram mergulhadas em solução de ácido cítrico $(1,0 \mathrm{~g}$ para $5,0 \mathrm{~L})$ a $100{ }^{\circ} \mathrm{C}$, por 2,5 minutos e imediatamente resfriadas em água gelada.

Preparação das amostras: as frutas foram cortadas em fatias, sendo as dimensões médias apresentadas na Tabela 1. Cabe ressaltar que o processo de secagem de cada uma das frutas estudadas foi realizada considerando três diferentes temperaturas (constantes durante todo o procedimento experimental), a saber, 60 ${ }^{\circ} \mathrm{C}, 65{ }^{\circ} \mathrm{C}$ e $70{ }^{\circ} \mathrm{C}$. O uso de amostras "fatiadas" como material a ser desidratado, é a forma mais comum do tratamento de produtos em geral (AKPINAR, 2006, SINGH et al., 2014). Neste caso, deixa-se de considerar um fenômeno modelado com geometria complexa para tratar o fenômeno com geometria mais simples, geralmente, coordenadas cartesianas.

Como destacado por Panchariya et al. (2002), os modelos que descrevem o fenômeno de secagem podem ser classificados em três categorias, a saber, modelos teóricos, semi-teóricos e empíricos. A primeira categoria é empregada para o estudo da resistência interna de transferência de umidade, enquanto as outras duas categorias são consideradas para o estudo da resistência externa de transferência de umidade entre o ar e o produto (WHITAKER et al. 1969; HENDERSON, 1974). Cabe ressaltar que durante o processo de secagem foi realizada a movimentação das bandejas para que não haja variação na temperatura recebida por elas. $\mathrm{O}$ acompanhamento da redução do conteúdo de umidade das amostras, para cada amostra, foi realizado por pesagens em determinados instantes de tempo em balança digital com precisão de $\pm 0,01 \mathrm{~g}$ até atingir a umidade entre 15 e $25 \%$.
Os valores utilizados para a formulação do problema de otimização são valores médios das amostras consideradas. $\mathrm{Na}$ presente contribuição serão considerados para fins de aplicação os modelos semiteóricos para a caracterização da cinética de secagem em camada fina. É importante ressaltar que esta escolha se deve a melhor capacidade de previsão de resultados que estes apresentam em comparação com outros modelos (Singh et al., 2014).

Um dos primeiros modelos de secagem em camada fina foi proposto por Lewis (1921). Este deriva do modelo semi-teórico empregado para materiais higroscópicos porosos, que é análogo à lei de resfriamento de Newton. O modelo de Lewis é descrito como (LEWIS, 1921):

$$
M R=\frac{X-X_{e}}{X_{0}-X_{e}}=\exp (-k t)
$$

em que MR é a taxa de umidade - Moisture Ratio $\left(\mathrm{g}_{\text {sólido }}\right.$ seco $\left./ \mathrm{g}_{\text {sólido úmido }}\right), k$ é a constante de secagem $\left(\mathrm{h}^{-1}\right), t$ é o tempo de secagem (h). $X, X_{e}$ e $X_{0}$ representam o teor de umidade, a qualquer instante de tempo, a umidade de equilíbrio e a umidade inicial, respectivamente.

Os modelos teóricos são derivados a partir da segunda lei de difusão de Fick (Panchariya et al., 2002), enquanto os modelos semi-teóricos são geralmente derivados de modificações da segunda lei de Fick e da lei de resfriamento de Newton (Erbay; Icier, 2010). Posto isto, as frutas foram dispostas em bandejas (Figura 1) e estas inseridas em um secador de armário (Figura 2).

Tabela 1. Dimensões médias $(\mathrm{mm})$ das amostras consideradas neste trabalho.

\begin{tabular}{cccc}
\hline & $60{ }^{\circ} \mathrm{C}$ & $65^{\circ} \mathrm{C}$ & $70{ }^{\circ} \mathrm{C}$ \\
\hline Abacaxi & $84,1 \times 76,1 \times 9,0$ & $94,3 \times 90,0 \times 9,7$ & $87,1 \times 83,4 \times 8,9$ \\
\hline Banana & $30,2 \times 33,6 \times 5,2$ & $27,1 \times 26,1 \times 5,4$ & $31,0 \times 30,7 \times 4,5$ \\
\hline
\end{tabular}

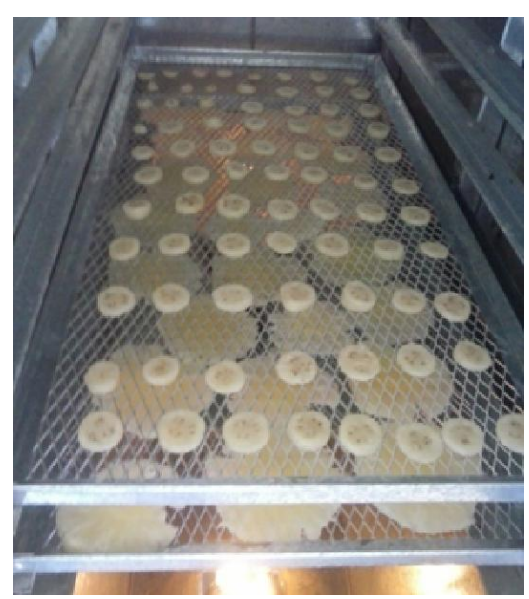

Figura 1. Frutas dispostas em bandejas no secador de armário. 


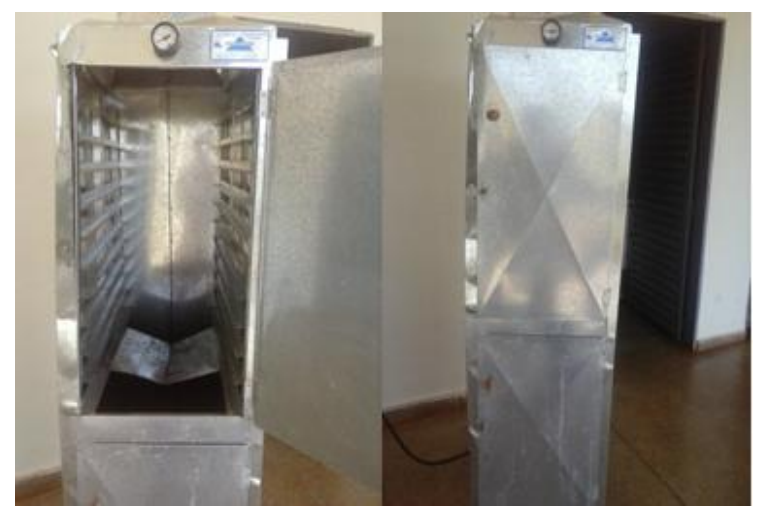

Figura 2. Secador do tipo armário utilizado para a secagem das frutas.

Shakari (2012) apresenta uma modificação do modelo proposto por Lewis (Equação de Page) através da adição de uma constante empírica adimensional $(n)$ :

$$
M R=\frac{X-X_{e}}{X_{0}-X_{e}}=\exp \left(-k t^{n}\right)
$$

Para a secagem de batata doce, Diamante e Munro (1993) propuseram uma modificação no Modelo de Page, sendo este dado pela seguinte equação (este é conhecido como Modelo de Page Modificado):

$$
M R=\frac{X-X_{e}}{X_{0}-X_{e}}=\exp \left(-k\left(t / l^{2}\right)^{n}\right)
$$

em que $l$ é uma constante empírica adimensional. Mais recentemente, Singh et al. (2014) propuseram, para a secagem de batata e maçã, o seguinte modelo:

$$
M R=\frac{X-X_{e}}{X_{0}-X_{e}}=\exp (-k t)-n t
$$

em que $k$ é a constante de secagem $\left(\mathrm{h}^{-1}\right)$ e $n$ é um parâmetro adimensional proposto para a obtenção de um melhor ajuste do modelo aos dados experimentais.

Os modelos acima apresentados serão testados para o ajuste dos dados experimentais obtidos conforme o procedimento descrito na seção anterior. Nas próximas seções são apresentados a formulação matemática do problema de ajuste de curvas (problema de otimização) e a metodologia empregada para a resolução do mesmo.

Como comentado anteriormente, a determinação dos parâmetros das equações empíricas apresentadas exige a formulação e resolução de um problema de otimização. Este consiste na minimização do funcional $F$, isto é, obter o valor do vetor de variáveis de projeto (parâmetros do modelo que deseja-se estimar) de modo a minimizar o somatório dos desvios quadráticos (distância entre os valores experimentais e os valores preditos pelo modelo proposto), conforme a seguinte equação:

$$
F \equiv \sum_{i=1}^{n_{\mathrm{exp}}}\left(y_{i}^{\exp }-y_{i}^{\mathrm{cal}}\left(\alpha_{1}, \alpha_{2}, \ldots, \alpha_{m}\right)\right)^{2}
$$

onde $y^{\text {cal }}$ e $y^{\text {exp }}$ representam o valor da variável dependente $(y)$ predito pelo modelo e o conjunto de pontos experimentais, respectivamente. $\alpha_{k}(k=1, \ldots, m)$ é o vetor que contém os $m$ parâmetros que devem ser determinados e $n_{\text {exp }}$ é o número de dados experimentais considerados no ajuste do modelo.

Para mensurar a qualidade do ajuste obtido com cada modelo, será utilizado o coeficiente de determinação $\left(r^{2}\right)$, definido como (CHAPRA, 2013):

$$
r^{2} \equiv 1-\frac{S_{r}}{S_{t}}
$$

em que $S_{r}$ (soma total dos desvios quadráticos entre os dados experimentais e os valores computados pelo modelo considerado) e $S_{t}$ (soma total dos quadrados dos resíduos entre os dados experimentais e a média) são definidos como:

$$
\begin{aligned}
& S_{r} \equiv \sum_{i=1}^{n_{\text {exp }}}\left(y_{i}^{\text {exp }}-y_{i}^{\text {cal }}\right)^{2} \\
& S_{t} \equiv \sum_{i=1}^{n_{\text {exp }}}\left(\bar{y}-y_{i}^{\text {cal }}\right)^{2}
\end{aligned}
$$

onde a média $(\bar{y})$ é definida como:

$$
\bar{y} \equiv \frac{\sum_{i=1}^{n_{\text {exp }}} y_{i}^{\text {cal }}}{n_{\text {exp }}}
$$

Em termos práticos, quanto mais próximo $r^{2}$ for da unidade, melhor é o ajuste proposto. Este valor representa o percentual dos dados experimentais que pode ser explicado pelo modelo matemático proposto. Neste caso, se o valor de $r^{2}$ for longe da unidade, isto implica que o modelo proposto não foi uma boa escolha (CHAPRA, 2013).

Os Algoritmos Genéticos (AG) são estratégias fundamentadas nos princípios de seleção natural postulados por Darwin (1859) para fins de resolução de problemas de otimização. Estes destacam que as populações existentes na natureza evoluem para garantir a sua própria sobrevivência (FILITTO, 2008).

Os primeiros estudos envolvendo os AG inicializaram-se com John Holland em meados anos 70, com a pesquisa sobre algoritmos que manipulavam strings de 0 e 1 , os denominados cromossomos. Este estudo resultou, em 1975, na publicação do livro Adaptation in Natural and Artificial Systems, considerado como a Bíblia dos Algoritmos Genéticos (FILITTO, 2008). Mas foi somente em 1989 com o estudo de David E. Goldberg que os AG se tornaram populares entre os pesquisadores da área de otimização (MICHALEWICZ, 1996). Estes algoritmos de natureza global fazem uso de uma estratégia de busca paralela e estruturada, mas aleatória, que é voltada em direção ao reforço da busca de pontos com melhor valor (em termos do valor da função objetivo). Apesar de aleatórios, pode-se dizer que a busca pelo ótimo não representa um conjunto de tentativas puramente aleatórias, mas de certa forma direcionadas, 
pois exploram informações baseadas no histórico para encontrar novos candidatos à solução do problema de otimização (MICHALEWICZ, 1996). De forma geral, a atualização da população de candidatos é realizada através de um procedimento iterativo. Durante cada geração, os princípios de reprodução, mutação e seleção são aplicados de modo que esta possa ser atualizada, como acontece na natureza (as espécies estão em constante evolução). A Figura 3 apresenta o fluxograma que apresenta os operadores nos AG.

A seguir é apresentada uma breve descrição de cada um dos operadores dos AG, bem como é realizada a sua inicialização.

Como em qualquer algoritmo evolutivo, a população é inicializada aleatoriamente (onde cada variável de projeto é delimitada pelo domínio especificado pelo usuário). Para essa finalidade, deve-se definir o tamanho da população, bem como a função objetivo, o número de variáveis de projeto e o respectivo domínio para que a população possa ser definida.

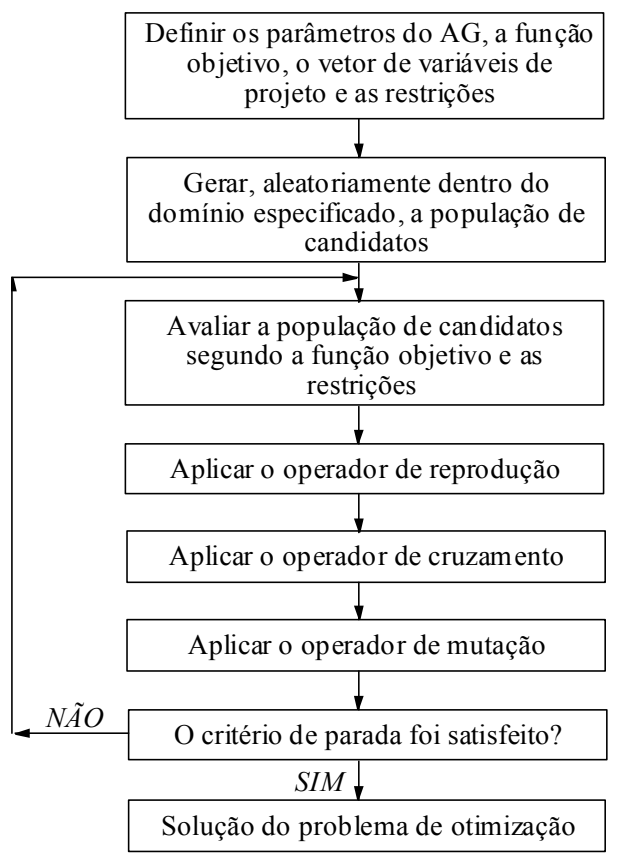

Figura 3.Fluxograma que apresenta os operadores nos AG. Fonte: Adaptado de Saramago (1999).

Por sua vez, o Operador de Reprodução tem como objetivo escolher os elementos da população que participarão do processo de reprodução, isto é, selecionar os pais dos indivíduos que estarão presentes na nova população. Esta escolha é realizada segundo a avaliação de cada candidato quanto à função objetivo. $\mathrm{O}$ candidato que apresenta o maior valor, em termos da função objetivo, tem a maior chance de contribuir à geração seguinte (com pelo menos um descendente). Assim, quanto maior o valor da função objetivo, maiores são as chances do candidato sobreviver no ambiente e reproduzir-se passando parte de seu material genético a gerações posteriores (SARAMAGO, 1999).

Neste caso, define-se a probabilidade relacionado com o i-ésimo candidato (pi) como sendo:

$$
p_{i} \equiv \frac{f_{i}(x)}{\sum_{j=1}^{n_{\text {var }}} f_{j}(x)}
$$

em que $n_{\text {var }}$ é o número de variáveis de projeto. Assim, se o candidato for de baixa adequabilidade, este tem alta probabilidade de desaparecer da população. Por outro lado, o candidato for de alta adequabilidade, o mesmo tem grandes chances de permanecer na população.

As probabilidades acumulativas $\left(q_{i}, i=1, \ldots, n_{\text {var }}\right)$ são definidas como:

$$
q_{i} \equiv \sum_{j=1}^{i} p_{j}
$$

A seguir deve-se selecionar as cadeias que irão contribuir para a geração seguinte. Esta seleção considera um conjunto de números $r$, escolhidos aleatoriamente entre $[0,1]$, em quantidade igual ao número de cadeias. A análise é feita através das seguintes opções:

- Se $r$ é menor que $q_{i}$, então seleciona-se o $i$-ésimo cromossomo.

- Por outro lado, se $r$ é maior que $q_{i}$, então analisar o subsequente cromossomo, isto é, $i+1$.

Vale ressaltar que alguns cromossomos poderão ser selecionados mais de uma vez, isto é, os melhores serão copiados mais vezes, enquanto que os de menor probabilidade poderão ser eliminados da população (SARAMAGO, 1999).

O operador de cruzamento consiste de troca de material genético de modo a promover a diversidade da população. Na literatura, inúmeras são as formas para se obter o cruzamento nos AG. Neste estudo será adotado o seguinte procedimento. Seja um ponto $k$ que define a posição de cruzamento na cadeia de bits de cada cromossomo escolhido aleatoriamente na população. A quantidade de cromossomos a ser submetida ao processo de cruzamento é definida através da probabilidade de cruzamento $p_{c}$ (especificada pelo usuário entre $[0,1]$, geralmente $25 \%$ ). Cada cadeia é quebrada no $k$-ésimo ponto e todas as informações do cromossomo $i$, a partir do ponto escolhido, são copiadas para o cromossomo $j$ e vice-versa, conforme esquematizado na Figura 4.

$$
\begin{aligned}
& \text { Antes do cruzamento Depois do cruzamento }
\end{aligned}
$$

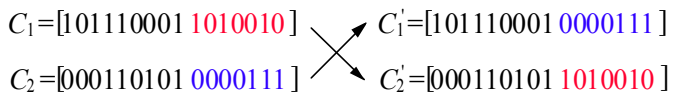

Figura 4. Representação esquemática do operador de cruzamento. Fonte: Adaptado de Saramago (1999). 
O processo de escolha de quem será compartilhado deve ser feito em pares, através da escolha via a geração de números aleatórios $\left(r_{i}\right)$. Quando não for possível formar os pares um novo sorteio deverá ser feito até obter os pares necessários para o cruzamento. Por exemplo, se $r_{1}$ for menor que $p_{c}$, então o primeiro cromossomo será selecionado. Após de ter feito isso, deve-se gerar um novo número aleatório para determinar a posição $k$ onde duas novas cadeias são formadas pela troca de todos os caracteres dos dois cromossomos selecionados. O operador de mutação consiste em uma modificação aleatória no valor de um alelo (que forma a variável de projeto em codificação binária) da cadeia (que forma o gene e, por consequência, o cromossomo). Caso o alelo escolhido seja zero passa a ser um e vice-versa, conforme esquematizado na Figura 5.

Antes da mutação
$C_{1}=[1001000100000001] \quad C_{1}^{\prime}=[1001000101000001]$

Figura 5. Representação esquemática do operador de mutação Fonte: Adaptado de Saramago (1999).

Em termos práticos, este operador consiste na seleção aleatória de uma posição em um cromossomo, a qual deve ser comparada com a probabilidade de mutação $p_{m}$ (escolhida pelo usuário como sendo geralmente igual a 1\%). Neste caso, se for gerado um número aleatório entre 0 e 1 e este for menor que a probabilidade de mutação, será realizada a mudança no bit do cromossomo, caso contrário, o cromossomo permanece sem nenhuma modificação. Este operador tem um papel importante e necessário, porque a aplicação dos operadores de reprodução e de cruzamento podem resultar na perda de material genético potencialmente útil (MICHALEWICZ, 1996).

\section{Resultados e Discussão}

Para aplicação da metodologia deste trabalho, alguns pontos devem ser destacados. Foram analisados dois produtos, a saber, abacaxi e banana. Para cada um destes foram levantados pontos experimentais, como descrito no item Material e Métodos, considerando as temperaturas de $60{ }^{\circ} \mathrm{C}, 65^{\circ} \mathrm{C}$ e $70{ }^{\circ} \mathrm{C}$ para a formulação da função objetivo a ser minimizada.

Foram analisadas quatro equações que representam a cinética de secagem [Equações (1) à (4)], e que apresentam diferentes números de parâmetros que devem ser estimados. Cabe ressaltar que para essa finalidade, os termos $X$ e $X_{0}$ foram considerados muito mais relevantes do que o termo $X_{e}$, isto é:

$$
\frac{X-X_{e}}{X_{0}-X_{e}} \approx \frac{X}{X_{0}}
$$

Parâmetros utilizados nos AG (SARAMAGO, 1999): população com 25 indivíduos, número de gerações igual a 250 , probabilidade de cruzamento igual a 0,25 e probabilidade de mutação igual a 0,1 . É importante ressaltar que, para os parâmetros considerados, são necessárias $25+25 \times 250$ avaliações da função objetivo em cada execução do algoritmo. Cada um dos estudos de caso foram simulados dez vezes com o seguinte vetor de sementes iniciais para o gerador de números aleatórios ([ $\left[\begin{array}{llll}0 & 1 & \ldots\end{array}\right]$.

O critério de parada adotado neste trabalho foi o número máximo de gerações, isto é, o procedimento evolutivo é finalizado se esse valor é alcançado. De posse dos dados experimentais obtidos com a secagem considerando cada temperatura, os modelos que representam a cinética de secagem para o abacaxi podem ser estimados. A Tabela 2 apresenta os resultados obtidos para a secagem do abacaxi considerando diferentes modelos e diferentes temperaturas. Observa-se que, primeiramente, o otimizador empregado foi capaz de estimar satisfatoriamente $\mathrm{o}(\mathrm{s})$ parâmetro(s) considerados para a cinética de secagem, quando avalia-se os valores da função objetivo para cada um. De forma geral, quanto maior o número de parâmetros do modelo, melhor tente a ser o ajuste, já que quanto maior o número de graus de liberdade, melhor o modelo se adapta aos pontos experimentais, conforme os valores obtidos para o coeficiente de determinação.

Após a realização do procedimento experimental, a Figura 6 apresenta o aspecto final do abacaxi desidratado. Nesta figura percebe-se que o objetivo da desidratação foi alcançado quando compara-se com a fruta in natura (Figura 1).

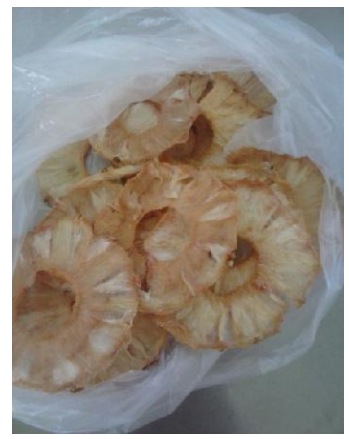

Figura 6. Desidratação do abacaxi após a secagem.

Todavia, é importante ressaltar, para o modelo representado pela Equação (3) e que apresenta três parâmetros, que não foi observado uma melhora significativa pelo uso de mais parâmetros em comparação com os modelos com dois parâmetros (Equações (2) e (4)). Assim, para este estudo de caso, o uso de um modelo com mais parâmetros não resultou na melhora do valor da função objetivo, isto é, tanto faz usar dois ou três parâmetros. 
Tabela 2. Parâmetros estimados para a cinética de secagem do abacaxi considerando diferentes modelos e temperaturas.

\begin{tabular}{|c|c|c|c|c|c|c|}
\hline \multirow{2}{*}{ Equação } & \multirow{2}{*}{$\mathrm{T}\left({ }^{\circ} \mathrm{C}\right)$} & \multicolumn{3}{|c|}{ Parâmetros } & \multirow{2}{*}{$F(-)$} & \multirow{2}{*}{$r^{2}$} \\
\hline & & $k\left(\mathrm{~h}^{-1}\right)$ & $n(-)$ & $l(-)$ & & \\
\hline \multirow{4}{*}{ (1) } & \multirow{2}{*}{60} & $0,330268^{1}$ & \multirow[b]{2}{*}{ - } & \multirow[b]{2}{*}{ - } & $0,005306^{1}$ & \multirow{2}{*}{$0,991503^{1}$} \\
\hline & & $1,530 \mathrm{E}-10^{2}$ & & & $2,258 \mathrm{E}-19^{2}$ & \\
\hline & \multirow{2}{*}{65} & 0,375343 & \multirow{2}{*}{ - } & \multirow[b]{2}{*}{ - } & 0,004242 & \multirow{2}{*}{0,993073} \\
\hline & & $1,507 \mathrm{E}-10$ & & & $5,007 \mathrm{E}-19$ & \\
\hline & \multirow{2}{*}{70} & 0,485637 & \multirow{2}{*}{ - } & \multirow{2}{*}{ - } & 0,005536 & \multirow{2}{*}{0,991539} \\
\hline & & $2,044 \mathrm{E}-10$ & & & $8,67 \mathrm{E}-19$ & \\
\hline & \multirow{2}{*}{60} & 0,284942 & 1,154924 & \multirow{2}{*}{ - } & 0,000374 & \multirow{2}{*}{0,999476} \\
\hline & & 0,086940 & 1,223884 & & 0,107056 & \\
\hline \multirow{6}{*}{ (2) } & \multirow{2}{*}{65} & 0,343374 & 1,083867 & \multirow[b]{2}{*}{ - } & 0,003257 & \multirow{2}{*}{0,994946} \\
\hline & & 0,002088 & 0,005177 & & $1,144 \mathrm{E}-5$ & \\
\hline & \multirow{2}{*}{70} & 0,432607 & 1,163522 & \multirow{2}{*}{-} & 0,000560 & \multirow{2}{*}{0,999241} \\
\hline & & 23,892841 & 2,334326 & & 0,285681 & \\
\hline & \multirow{2}{*}{60} & 0,721770 & 1,154926 & 1,495369 & 0,000371 & \multirow{2}{*}{0,999476} \\
\hline & & 34,126866 & 41,141953 & 3,883489 & 0,262558 & \\
\hline \multirow{4}{*}{ (3) } & \multirow{2}{*}{65} & 3,400907 & 1,083879 & 2,879935 & 0,003257 & \multirow{2}{*}{0,994946} \\
\hline & & 49,655311 & 31,667967 & 10,253508 & 0,162355 & \\
\hline & & 1,586328 & 1,163522 & 1,747817 & 0,000560 & \\
\hline & 70 & 32,508483 & 34,571715 & 3,112043 & 0,197511 & 0,999241 \\
\hline & 60 & 0,278609 & 0,019190 & & 0,001526 & 0007783 \\
\hline & 60 & 0,088257 & 0,053679 & - & 0,013371 & $0,99 / / 83$ \\
\hline & & 0,372876 & 0,000633 & & 0,004233 & \\
\hline (4) & 65 & $1,063 \mathrm{E}-4$ & $2,203 \mathrm{E}-5$ & - & $2,575 \mathrm{E}-8$ & 0,993115 \\
\hline & 70 & 0,413757 & 0,022358 & & 0,001427 & רתקס000 \\
\hline & 10 & $9,920 \mathrm{E}-4$ & $2,997 \mathrm{E}-4$ & - & $1,709 \mathrm{E}-6$ & $0,9980 \angle 2$ \\
\hline
\end{tabular}

${ }^{T}$ Melhor solução e ${ }^{2}$ Desvio padrão computados a partir das 10 execuções do algoritmo.

Para o modelo representado pela Equação (4), com dois parâmetros, para a temperatura de $65^{\circ} \mathrm{C}$, observase que os parâmetros obtidos $\left(k=0,372876 \mathrm{~h}^{-1}\right.$ e $n=0,0006333)$ tendem ao encontrado pelo representado pela Equação (1), com um único parâmetro, isto é, $k=0,375343 \mathrm{~h}^{-1}$ e $n=0$. Isto implica que, para esta configuração, os dois modelos são equivalentes. Finalmente, ressalta-se, para alguns estudos de caso [obtidos pela aplicação das Equações (2), (3) e (4)], que os AG obtiveram, em cada uma das dez execuções consideradas, valores diferentes para a função objetivo, conforme o valor do desvio padrão apresentado nesta tabela. Em termos da otimização, isto pode implicar na obtenção de ótimos locais ou que, para a configuração empregada nos AG, este não foi capaz de melhorar o valor da função objetivo. Apesar disto, considera-se que a melhor solução possível, considerando a configuração empregada nos AG, foi obtida.

A Figura 7 apresenta a comparação entre os pontos experimentais e estimados por cada um dos modelos cinéticos para o abacaxi considerando diferentes temperaturas. Observa-se boa concordância entre os pontos experimentais e os simulados com os parâmetros obtidos pelos AG.

$\mathrm{Na}$ figura 7 observa-se que quanto maior a temperatura, mais rápido o produto perde massa e é desidratado. Do ponto de vista físico, este resultado já era esperado, conforme enfatizado por diversos autores (OVERHULT et al., 1973; PANCHARIYA et al., 2002). Analogamente ao observado experimentalmente para o abacaxi, na Figura 8 é apresentado o aspecto final da banana desidratada com o procedimento experimental adotado.

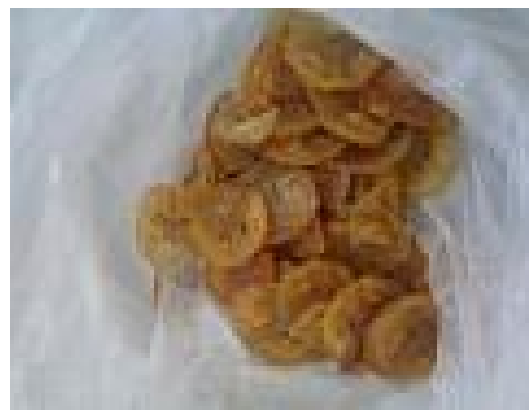

Figura 8.Desidratação da banana após a secagem.

Com os dados experimentais obtidos, a cinética de secagem da banana pode ser estimada. A Tabela 3 apresenta os resultados obtidos considerando diferentes modelos e diferentes temperaturas. Como constatado no estudo de caso anterior, observa-se nesta tabela o método de otimização empregado foi capaz de estimar satisfatoriamente $\mathrm{o}(\mathrm{s})$ parâmetro(s) considerados para a cinética de secagem da banana. Percebe-se que os 
modelos representados pelas Equações (2) e (3) são equivalentes, isto é, o aumento do número de parâmetros não resultou na melhora do valor da função

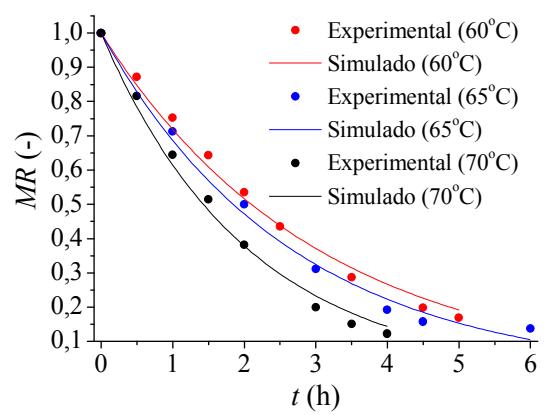

(a) Modelo de Lewis - Equação (1).

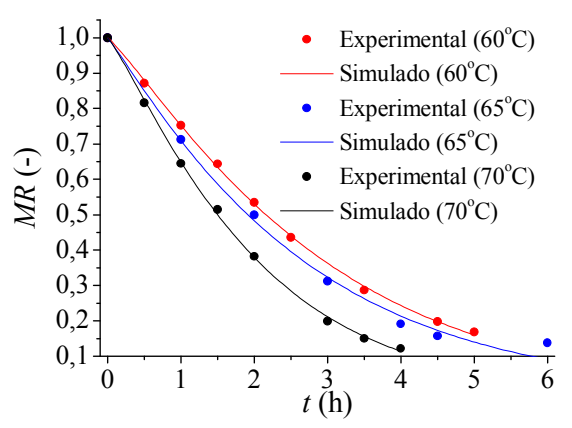

(c) Modelo de Page Modificado - Equação (3). objetivo. Chega-se a mesma conclusão quando avalia-se a qualidade do ajuste, em termo da função objetivo e do coeficiente de determinação, para as Equações (1) e (4).

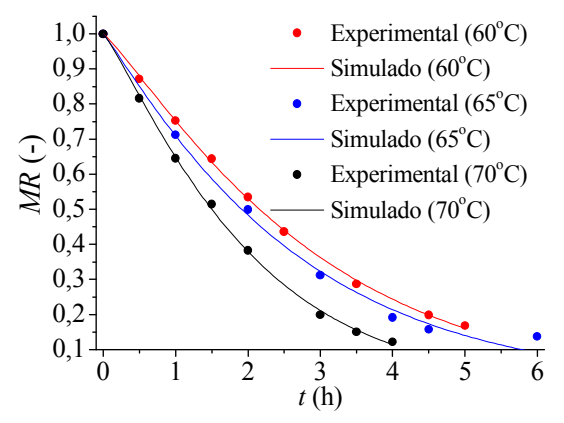

(b) Modelo de Page - Equação (2).

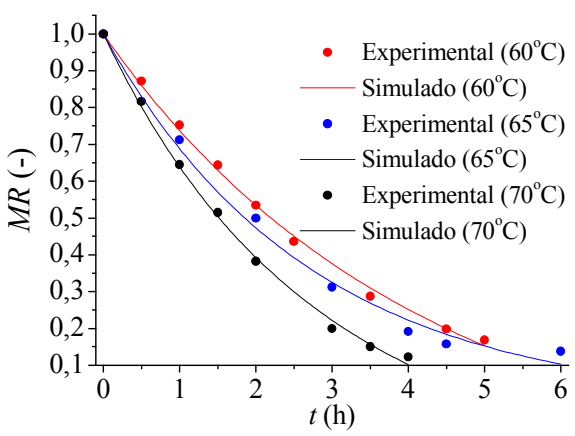

(d) Modelo de Singh - Equação (4).

Figura 7. Comparação entre os pontos experimentais e estimados por cada um dos modelos cinéticos para o abacaxi.

Tabela 3. Parâmetros estimados para a cinética de secagem da banana considerando diferentes modelos e temperaturas.

\begin{tabular}{|c|c|c|c|c|c|c|}
\hline \multirow{2}{*}{ Equação } & \multirow{2}{*}{$\mathrm{T}\left({ }^{\circ} \mathrm{C}\right)$} & \multicolumn{3}{|c|}{ Parâmetros } & \multirow{2}{*}{$F(-)$} & \multirow{2}{*}{$r^{2}$} \\
\hline & & $k\left(\mathrm{~h}^{-1}\right)$ & $n(-)$ & $l(-)$ & & \\
\hline \multirow{3}{*}{ (1) } & 60 & $\begin{array}{c}0,386307^{1} \\
3,855 \mathrm{E}-10^{2}\end{array}$ & - & - & $\begin{array}{c}0,030399^{1} \\
1,989 \mathrm{E}-18^{2}\end{array}$ & $0,956331^{1}$ \\
\hline & 65 & $\begin{array}{c}0,386810 \\
4,198 \mathrm{E}-10\end{array}$ & - & - & $\begin{array}{c}0,075775 \\
4,625 \mathrm{E}-18\end{array}$ & 0,899302 \\
\hline & 70 & $\begin{array}{c}0,595244 \\
4,662 \mathrm{E}-10\end{array}$ & - & - & $\begin{array}{c}0,045944 \\
6,542 \mathrm{E}-18\end{array}$ & 0,937012 \\
\hline \multirow{3}{*}{ (2) } & 60 & $\begin{array}{c}0,476325 \\
23,906029\end{array}$ & $\begin{array}{l}0,748566 \\
2,462941\end{array}$ & - & $\begin{array}{l}0,011780 \\
0,359242\end{array}$ & 0,978427 \\
\hline & 65 & $\begin{array}{l}0,698985 \\
0,000875\end{array}$ & $\begin{array}{l}0,488165 \\
0,001007\end{array}$ & - & $\begin{array}{l}0,009684 \\
7,683 \mathrm{E}-7\end{array}$ & 0,981917 \\
\hline & 70 & $\begin{array}{c}0,722539 \\
30,463385 \\
\end{array}$ & $\begin{array}{l}0,643294 \\
2,343184 \\
\end{array}$ & - & $\begin{array}{l}0,012062 \\
0,173850 \\
\end{array}$ & 0,977688 \\
\hline \multirow{3}{*}{ (3) } & 60 & $\begin{array}{c}0,751177 \\
33,469470\end{array}$ & $\begin{array}{c}0,748566 \\
31,234136\end{array}$ & $\begin{array}{l}1,355642 \\
6,132795\end{array}$ & $\begin{array}{l}0,011780 \\
0,340293\end{array}$ & 0,978428 \\
\hline & 65 & $\begin{array}{l}61,602848 \\
36,488655\end{array}$ & $\begin{array}{c}0,488166 \\
28,994386\end{array}$ & $\begin{array}{l}98,238855 \\
30,689216\end{array}$ & $\begin{array}{l}0,009684 \\
0,202979\end{array}$ & 0,981918 \\
\hline & 70 & $\begin{array}{c}1,229608 \\
33,707343 \\
\end{array}$ & $\begin{array}{c}0,643294 \\
43,195063\end{array}$ & $\begin{array}{c}1,511718 \\
31,270875 \\
\end{array}$ & $\begin{array}{l}0,012062 \\
0,221854\end{array}$ & 0,977688 \\
\hline \multirow{3}{*}{ (4) } & 60 & $\begin{array}{c}0,386306 \\
1,728 \mathrm{E}-10 \\
\end{array}$ & $\begin{array}{l}0 \\
0 \\
\end{array}$ & - & $\begin{array}{c}0,030399 \\
1,635 \mathrm{E}-18 \\
\end{array}$ & 0,956331 \\
\hline & 65 & $\begin{array}{c}0,386810 \\
2,558 \mathrm{E}-10\end{array}$ & $\begin{array}{l}0 \\
0\end{array}$ & - & $\begin{array}{c}0.075775 \\
6,542 \mathrm{E}-18\end{array}$ & 0,899302 \\
\hline & 70 & $\begin{array}{c}0,595244 \\
7,142 \mathrm{E}-10\end{array}$ & $\begin{array}{l}0 \\
0\end{array}$ & - & $\begin{array}{c}0,045944 \\
4,625 \mathrm{E}-18\end{array}$ & 0.937012 \\
\hline
\end{tabular}

${ }^{1}$ Melhor solução e ${ }^{2}$ Desvio padrão computados a partir das 10 execuções do algoritmo.

Revista de Agricultura Neotropical, Cassilândia-MS, v. 3, n. 3, p. 28-37, jul./set. 2016. 
Para todas as temperaturas, os resultados obtidos pelo modelo com dois parâmetros são equivalentes para o modelo com um único parâmetro (Equação (1)), já que todos os valores obtidos para o parâmetro $n$ foram iguais a zero. Conforme parâmetros para a cinética de secagem do abacaxi, para alguns estudos de caso (obtidos pela aplicação das Equações (2) e (3)), os AG obtiveram, em cada uma das dez execuções consideradas, valores diferentes para a função objetivo, conforme o valor do desvio padrão apresentado na tabela abaixo. Este resultado pode representar a obtenção de ótimos locais ou que, para a configuração empregada nos AG, este não foi capaz de melhorar o valor da função objetivo. Apesar disto, para a configuração empregada nos AG, considera-se que a melhor solução possível foi alcançada.

A Figura 9 apresenta a comparação entre os pontos experimentais e estimados por cada um dos modelos cinéticos para a banana considerando diferentes temperaturas.

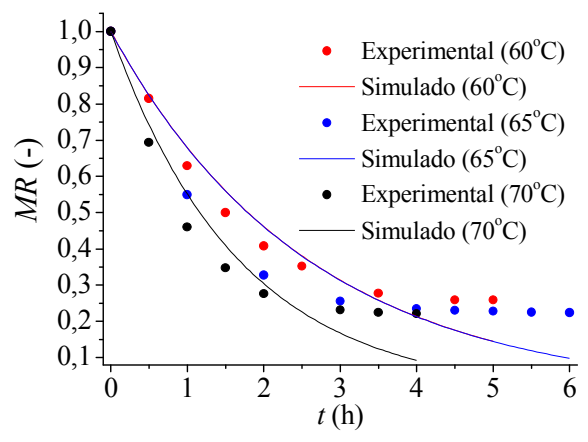

(a) Modelo de Lewis - Equação (1).

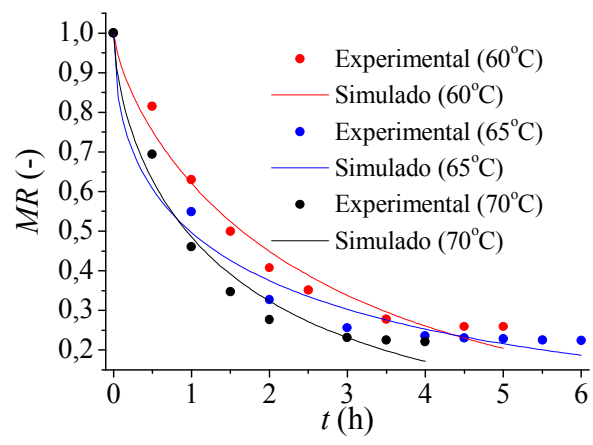

(c) Modelo de Page Modificado - Equação (3).

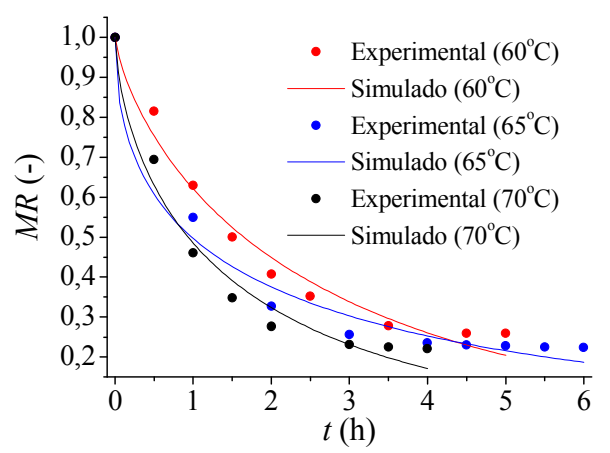

(b) Modelo de Page - Equação (2).

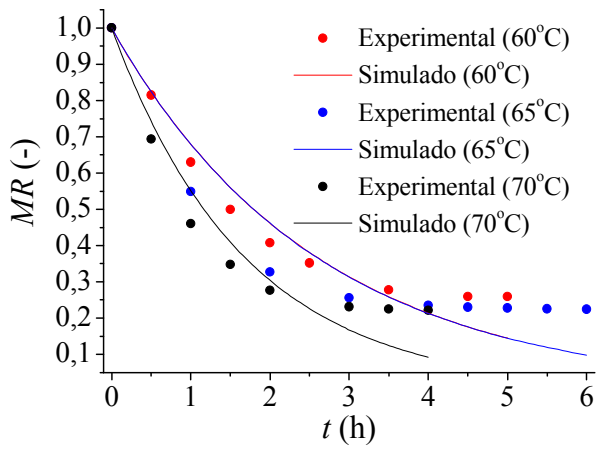

(d) Modelo de Singh - Equação (4).

Figura 9. Comparação entre os pontos experimentais e estimados por cada um dos modelos cinéticos para a banana.

\section{Conclusões}

Este trabalho experimental-computacional teve por objetivo a determinação da cinética de secagem de produtos alimentícios através da formulação e resolução de um problema de otimização. Para essa finalidade foram obtidos pontos experimentais para dois produtos,a saber, para o abacaxi e para a banana, considerando diferentes temperaturas. Os parâmetros das equações cinéticas consideradas foram estimados usando os Algoritmos Genéticos com diferentes sementes iniciais para a obtenção dos valores médios e dos desvios padrão apresentados nas tabelas. De forma geral observa-se, para os estudos de caso analisados, que a metodologia apresentada configurou-se como uma alternativa interessante para a resolução do problema proposto, já que foram obtidos resultados considerados satisfatório, visto o valor da função objetivo e do coeficiente de determinação. Os melhores ajustes foram obtidos para as equações que apresentam apenas dois parâmetros. Foi constatado, para os estudos de caso analisados, que o aumento do número de parâmetros não implicou na melhora do valor da função objetivo.

Como continuidade de trabalho propõe-se estimar os parâmetros das equações cinéticas de secagem considerando a inserção de robustez e de confiabilidade ao problema de otimização original.

\section{Agradecimentos}

Os autores agradecem a FAPEMIG, ao CNPq e a CAPES pelo suporte financeiro deste trabalho. 


\section{Referências Bibliográficas}

AKPINAR, E. K. Determination of suitable thin layer drying curve model for some vegetables and fruits. Journal of Food Engineering, London-United Kingdom, v. 73, n. 12, p. 75-84, 2006.

ARRUDA, E. B.; LOBATO, F. S.; ASSIS, A. J.; BARROZO, M. A. S. Modeling of fertilizer drying in roto-aerated and conventional rotary dryers. Drying Technology, LondonUnited Kingdom, v. 27, n. 3, p. 1192-1198, 2009.

CEYLAN, I.; AKTAS, M.; DOGAN, H., Mathematical modeling of drying characteristics of tropical fruits. Applied Thermal Engineering. London-United Kingdom, v. 27, n. 4, p. 1931-1936, 2007.

CHAPRA, S. C. Métodos numéricos aplicados com Matlab para engenheiros e cientistas. $3^{\mathrm{a}}$ ed. Editora Mc Graw Hill, New York-USA, 2013, 655 p.

DARWIN, C. On the origin old species: by means of natural selection or the preservation of favoured races in the struggle for life. London, 1859, $1028 \mathrm{p}$.

DIAMANTE, L. M.; MUNRO, P. A. Mathematical modelling of the thin layer solar drying of sweet potato slices. Solar Energy. London-United Kingdom, v. 51, n. 4, p. 271-276, 1993.

ERBAY, Z.; ICIER, F. A review of thin layer drying of foods: theory, modeling, and experimental results. Critical Reviews in Food Science and Nutrition, London-United Kingdom, v. 50, n. 134, p. 441-464, 2010.

FARIA, R. Q.; TEIXEIRA, I. R; DEVILLA, I. A.; ASCHERI, D. P. R.; RESENDE, O. Cinética de Secagem de sementes de crambe. Revisa Brasileira de Engenharia Agrícola e Ambiental, Campina Grande-PB, v. 16, n. 3, p. 573-583, 2012.

FILITTO, D. Algoritmos genéticos: uma visão explanatória. Revista Multidisciplinar da Uniesp - Saber Acadêmico, São Paulo-SP, v. 6, n.3, p. 136-143, 2008.

HENDERSON, S. M. Progress in developing the thin layer drying equation. Transactions of ASAE, St. Joseph-USA, v. 17, n. 4, p. 1167-1172, 1974.

KEMP, I. C.; OAKLEY, D. E. Modeling of particulate drying in theory and practice. Drying Technology, London-United Kingdom, v. 20, n. 45, p. 1699-1750, 2002.

KUMAR, C.; KARIM, A.; SAHA, S. C.; JOARDDER, M. U. H.; BROWN, R.; BISWAS, D. Multiphysics modelling of convective drying of food materials. Proceedings of the Global Engineering, Science and Technology Conference, Dhaka, Bangladesh, 2012.

LEWIS, W. K. The rate of drying of solid materials. Journal of Industrial \& Engineering Chemistry, London-United Kingdom, v. 13, n. 5, p. 427-432, 1921.

LOBATO, F. S.; ARRUDA, E. B.; BARROZO, M. A. S.; STEFFEN JR, V. Estimation of drying parameters in rotary dryers using differential evolution. Journal of Physics Conference Series, New York-USA, v. 135, n. 1, p. 1-8, 2008.
MICHALEWICZ, Z. Genetic algorithms + data structure = evolution programs. $3^{\text {rd }}$ ed. Berlin: Springer, 1996, $568 \mathrm{p}$.

MONTEIRO, R. L.; A. M. B., CARCIOFI; LAURINDO, J. B. A microwave multi-flash drying process for producing crispy bananas. Journal of Food Engineering, London-United Kingdom, v. 178, n. 4, p. 1-11, 2016

OVERHULTS, D. G.; WHITE, G. M.; HAMILTON, H. E.; ROSS, I. J. Drying soybeans with heated air. Transactions of the ASAE, New York-USA, v. 16, n. 3, p. 112-113, 1973.

PAGE, G. E. Factors influencing the maximum rate of air drying shelled corn in thin-layers. M. S. Thesis, Purdue University, West Lafayette, Indiana, 1949.

PANCHARIYA, P. C.; POPOVIC, D.; SHARMA, A. L. Thinlayer modelling of black tea drying process. Journal of Food Engineering, London-United Kingdom, v. 52, n.4, p. 349$357,2002$.

SARAMAGO, S. F. P. Métodos de otimização randômica: algoritmos genéticos e simulated annealing, Notas em Matemática Aplicada, v. 6, São Carlos-SP: SBMAC, 1999, 35 p.

SHAHARI, N. A., Mathematical modelling of drying food products: application to tropical fruits. Nottingham: University of Nottingham, 2012, 247 p.

SINGH, F.; KATIYAR, V. K.; SINGH, B. P. Mathematical modeling to study drying characteristic of Apple and potato. International Conference on Chemical, Environment \& Biological Sciences (CEBS-2014), Kuala Lumpur (Malaysia), 2014 .

TADINI, C. C.; TELIS, V. R. N.; MEIRELLES, A. J. A.; PESSOA-FILHO, P. A. Operações unitárias na indústria de alimentos. v. 2 São Paulo-SP: Editora LTC, 2016, 652 p.

UDOMKUN, P.; ARGYROPOULOS, D.; NAGLE, M.; MAHAYOTHEE, B.; JANJAI, S.; MÜLLER, J. Single layer drying kinetics of papaya amidst vertical and horizontal airflow. LWT - Food Science and Technology, LondonUnited Kingdom, v. 64, n. 23, p. 67-73, 2015.

WHITAKER, T.; BARRE, H. J.; HAMDY, M. Y. Theoretical and experimental studies of diffusion in spherical bodies with a variable diffusion coefficients. Transactions of ASAE, New York-USA, v. 11, n. 2, p. 668-672, 1969.

WHITE, G. M.; BRIDGES, T. C.; LOEWER, O. J.; ROSS, I. J. Seed coat damage in thin layer drying of soybeans as affected by drying conditions. ASAE Journal, New YorkUSA, v. 34, n. 3052, 1978.

ZLATANOVIC, I; KOMATINA M.; ANTONIJEVIC, D. Low-temperature convective drying of apple cubes. Applied Thermal Engineering, London-United Kingdom, v. 53, n. 1, p. 114-123, 2013 